\title{
Are improper kinetic models hampering drug development?
}

Reproducibility of biological data is a significant problem in research today. One potential contributor to this, which has received little attention, is the over complication of enzyme kinetic inhibition models. The over complication of inhibitory models stems from the common use of the inhibitory term $\left(1+[I] / K_{i}\right)$, an equilibrium binding term that does not distinguish between inhibitor binding and inhibitory effect. Since its initial appearance in the literature, around a century ago, the perceived mechanistic methods used in its production have spurred countless inhibitory equations. These equations are overly complex and are seldom compared to each other, which has destroyed their usefulness resulting in the proliferation and regulatory acceptance of simpler models such as IC50s for drug characterization. However, empirical analysis of inhibitory data recognizing the clear distinctions between inhibitor binding and inhibitory effect can produce simple logical inhibition models. In contrast to the common divergent practice of generating new inhibitory models for every inhibitory situation that presents itself. The empirical approach to inhibition modeling presented here is broadly applicable allowing easy comparison and rational analysis of drug interactions. To demonstrate this, a simple kinetic model of DAPT, a compound that both activates and inhibits $\gamma$-secretase is examined using excel. The empirical kinetic method described here provides an improved way of probing disease mechanisms, expanding the investigation of possible therapeutic interventions. 


\section{Are improper kinetic models hampering drug development?}

2 Ryan Walsh*

3 Dept of Chemistry

4 Carleton University

5 Ottawa ON

6 rwalsh@connect.carleton.ca 
7 Reproducibility of biological data is a significant problem in research today. One potential

8 contributor to this, which has received little attention, is the over complication of enzyme kinetic

9 inhibition models. The over complication of inhibitory models stems from the common use of

10 the inhibitory term $\left(1+[\mathrm{I}] / \mathrm{K}_{\mathrm{i}}\right)$, an equilibrium binding term that does not distinguish between

11 inhibitor binding and inhibitory effect. Since its initial appearance in the literature, around a

12 century ago, the perceived mechanistic methods used in its production have spurred countless

13 inhibitory equations. These equations are overly complex and are seldom compared to each

14 other, which has destroyed their usefulness resulting in the proliferation and regulatory

15 acceptance of simpler models such as IC50s for drug characterization. However, empirical

16 analysis of inhibitory data recognizing the clear distinctions between inhibitor binding and

17 inhibitory effect can produce simple logical inhibition models. In contrast to the common

18 divergent practice of generating new inhibitory models for every inhibitory situation that presents

19 itself. The empirical approach to inhibition modeling presented here is broadly applicable

20 allowing easy comparison and rational analysis of drug interactions. To demonstrate this, a

21 simple kinetic model of DAPT, a compound that both activates and inhibits $\gamma$-secretase is

22 examined using excel. The empirical kinetic method described here provides an improved way

23 of probing disease mechanisms, expanding the investigation of possible therapeutic interventions.

\section{The problem with classical inhibition models}


25 Inhibitors bind to enzymes according to the same principles that govern ligand and receptor

26 interactions. That is enzyme inhibitors are subject to the same mass action kinetic principles used

27 to define the Hill-Langmuir equation and the Michaelis-Menten equation. However, the way

28 enzyme inhibition equations are currently produced suggests that kinetically enzyme inhibitor

29 interactions are as unique as the enzyme inhibitor system they are used to represent. This

30 problem stems from the supposed mechanistic derivation of the principle inhibition equations.

31 Mechanistic approaches resulted in an ambiguous inhibitory term that does not distinguish

32 between the stoichiometric enzyme inhibitor binding interactions, defined by the inhibition

33 constant $\left(\mathrm{k}_{\mathrm{i}}\right)$ and the effect the inhibitor has on the enzyme.

34 The principal inhibition equations competitive, non-competitive and mixed non-competitive

35 inhibition were produced using the reaction schemes depicted in Fig. 1 (McElroy, 1947). As can

36 be observed in the competitive inhibition reaction scheme (Fig. 1A), the equation results from the

37 blockade of enzyme substrate interactions by the inhibitor. This equation has been exclusively

38 used to describe a blockage produced by inhibitor binding to the active site of the enzyme.

39 Alternatively the non-competitive inhibition equation (Fig. 1B) is derived from a reaction scheme

40 where the inhibitor binds the enzyme substrate complex. The mixed non-competitive inhibition

41 equation (Fig. 1C) is used to describe inhibitors that can bind to the free enzyme or the enzyme

42 substrate complex.

43 When modeling the competitive inhibition equation an exclusive decrease in the substrate affinity

44 (increase in $\mathrm{K}_{1}$ value) is observed. In contrast, the non-competitive equation produces an

45 exclusive decrease in the maximum reaction rate $\left(\mathrm{V}_{1}\right)$, and the mixed non-competitive inhibition

46 equation describes inhibition where both substrate affinity and reaction rate are affected.

47 The flaws in the assumptions used to generate these inhibitory models are easy to identify and

48 has resulted in a field of scientific inquiry continually producing additional equations, to fill in 
49 the gaps. For example, changes in substrate affinity can result from mechanisms other than the

50 inhibitor binding to the active site. Kinetic characterization of mutant enzymes or the

51 comparisons of enzymes from different species has clearly demonstrated that substrate affinity

52 directly relates to the spatial conformation of the active site. So competitive inhibition that is

53 believed to be the predominant way in which inhibitors affect substrate affinity is not able to

54 account for inhibitor interactions that alter the spatial conformation of the active site. An

55 example of this false competitive inhibition is the inhibition of the peptidase kallikrein by

56 benzamidine (Sousa et al., 2001). Inhibition of kallikrein by benzamidine is known to occur

57 when benzamidine blocks the binding of peptide side chains but not the active site (Bernett et al.,

58 2002). This results in a decrease in the peptidases substrate affinity but not catalytic activity,

59 which can be conceptualized as the production of a new inhibitor derived surface which is less

60 favorable for substrate binding (Walsh, Martin \& Darvesh, 2011; Walsh, 2012).

61 The assumptions used to generate the non-competitive model only take into account complete

62 catalytic inhibition. A schematic representation of this is usually, depicted as an inhibitor binding

63 to the enzyme in a location other than the active site (Fig. 1), inducing a change that prevents

64 substrate hydrolysis. The main problem with this schematic representation is there is no way to

65 distinguish kinetically between non-competitive and competitive inhibition using these models.

66 In each case inhibitor binding would drive product formation to zero as inhibitors binding at the

67 active site or in a peripheral site would prevent enzymatic catalysis. Additionally the non-

68 competitive inhibition model fails to consider situations where the inhibitor may only partially

69 reduce the catalytic rate of the enzyme. Such effects can easily be rationalized by considering

70 situations where the inhibitor binds and does not affect the substrate binding site. This binding

71 may however alter the spatial conformation of the amino acids or cofactors involved in the

72 catalytic activity, reducing the efficiency of the enzymatic activity. 
73 The mixed non-competitive model, as a combination of the competitive and non-competitive

74 models, describes inhibition that affects substrate affinity and the catalytic rate of the enzyme.

75 However, based on the reaction schemes used to generate the competitive and non-competitive

76 inhibition models both one to one enzyme inhibitor binding events are already accounted for (Fig.

77 1). To rationalize this problem, the classical mechanistic theory allows mixed non-competitive

78 inhibitor to bind to their target with two different inhibition constants $\left(\mathrm{K}_{\mathrm{i}}\right.$ and $\left.\alpha \mathrm{K}_{\mathrm{i}}\right)$. This unusual

79 theory excludes the possibility an inhibitor could affect both substrate affinity and catalytic

80 activity in one enzyme inhibitor binding interaction.

81 The complexity only continues to grow from there, with the uncompetitive inhibition equation

82 that describes inhibitors that produce linear changes in both substrate affinities and catalytic rate

83 (Dodgson, Spenceret \& Williams, 1956). This unusual form of inhibition increases the substrate

84 affinity of the enzyme while decreasing activity and has been suggested to result from

85 interactions with multienzyme systems. Eventually, problems with these complete inhibitor

86 models began to be recognized, and a partial inhibition class of models occurred (Segel, 1975;

87 Yoshino, 1987). This complexity did not just affect the single substrate, single inhibitor

88 framework but was replicated in the development of multi-substrate and multi-inhibitor

89 frameworks (Segel, 1975). With the ever expanding base of mechanistic equations and the

90 almost unanimous ambivalence to them expressed by researcher, industry and regulatory bodies,

91 enzyme inhibitory characterization has diminished to the basic inhibitory equations or IC50s. In

92 response to this marginalization of mechanistic kinetic studies, there have been attempts to

93 reformulate the single substrate/inhibitor equations (Fontes et al., 2000). Unfortunately, they

94 have continued to embrace the same inhibitory terms that do not distinguish between binding

95 constants and inhibitory effect. 
96 One of the reasons that these models have endured for so long may be related to the false sense of

97 security; the equations for these models provide. The common form of the inhibitory term (1+

$98[\mathrm{I}] / \mathrm{K}_{\mathrm{i}}$ ) found in every equation suggests that it is working in a similar way. This notion is entirely

99 incorrect. The inhibitory term in the competitive inhibition equation (Fig. 1A) directly affects the

100 substrate affinity $\left(\mathrm{K}_{1}\right)$ by multiplying into it. The inhibitory term in the non-competitive

101 inhibition equation (Fig. 1B) inversely affects the maximum catalytic activity $\left(\mathrm{V}_{1}\right)$ by dividing

102 into it. A rearrangement of the non-competitive inhibition equation, demonstrates that the

103 described decrease in catalytic activity is directly dependent on an inhibitor binding term that is

104 identical to the mass action terms used to describe, ligand receptor interactions in the Hill-

105 Langmuir equation and substrate enzyme interactions in the Michaelis-Menten equation

106 (equation 1).

107

$$
v=V_{1} \frac{[S]}{\left([S]+K_{1}\right)\left(1+\frac{[I]}{K_{i}}\right)}=\frac{[S]}{[S]+K_{1}} V_{1} \times\left(1-\frac{[I]}{[I]+K_{i}}\right)=\frac{[S]}{[S]+K_{1}} \times\left(V_{1}-V_{1} \frac{[I]}{[I]+K_{i}}\right)
$$

108 The implications of this are that the non-competitive inhibition equation is the only one of these

109 three equations that is even somewhat correct. A similar rearrangement of the competitive

110 inhibition inhibitory term demonstrates that the change in substrate affinity is not directly related

111 to inhibitor binding. The change in substrate affinity $\left(\mathrm{K}_{1}\right)$ results from the substrate affinity term

112 being divided by the percent of the enzyme population that is not interacting with the inhibitor

113 (equation 2). This inversion of the inhibitor mass binding term is very similar to the inversion of

114 the Michaelis-Menten equation used in Lineweaver-Burk plots. This inversion linearizes the 
115 Michaelis-Menten equation and provides a graphical method of determining enzyme kinetic

116 constants. This linear relationship explains why the competitive inhibition equation can only be

117 used to describe linear increases in the substrate affinity. It also provides the rational as to why

118 the competitive inhibition equation is not useful for describing systems where the inhibitor

119 produces a finite hyperbolic shift in the substrate affinity.

$$
v=V_{1} \frac{[S]}{[S]+K_{1}\left(1+\frac{[I]}{K_{i}}\right)}=V_{1} \frac{[S]}{[S]+\frac{K_{1}}{\left(1-\frac{[I]}{[I]+K_{i}}\right)}}
$$

121 The linear relationship of the competitive inhibition equation and hyperbolic relationship

122 described by the non-competitive equation also suggests an explanation for the use of two

123 binding constants in the mixed non-competitive inhibition equation. Since the inhibitory terms of

124 these equations are affecting the constants of the Michaelis-Menten equation in different ways,

125 two inhibitor binding terms are required $\mathrm{K}_{\mathrm{i}}$ and $\alpha \mathrm{K}_{\mathrm{i}}$ (Fig. 1)(Walsh, 2012).

126 By tying inhibitory effects to mass action inhibitor binding terms, all three base inhibitory

127 equations and equations derived to explain situations they fail to cover, can be replaced with a

128 simple empirical relationship (equation 3) (Walsh, Martin \& Darvesh, 2011; Walsh, 2012).

$$
v=\frac{[S]}{[S]+K_{1}-\left(K_{1}-K_{1 i}\right) \frac{[I]}{[I]+K_{i}}} V_{1}-\left(V_{1}-V_{1 i}\right) \frac{[I]}{[I]+K_{i}}
$$


130 That is, virtually any inhibitor and enzyme interaction can be described as a shift in the catalytic

131 activity that is directly dependent on the mass binding of the inhibitor molecules to the enzyme

132 population being studied (Walsh, Martin \& Darvesh, 2011). The change induced by the inhibitor

133 can relate to substrate affinity $\left(\mathrm{K}_{1} \rightarrow \mathrm{K}_{1 \mathrm{i}}\right)$, maximum reaction velocity $\left(\mathrm{V}_{1} \rightarrow \mathrm{V}_{1 \mathrm{ii}}\right)$ or both. This

134 distinction between binding and effect provides an easy way of describing the vast spectrum of

135 changes in enzymatic activity that can result from the influence of inhibitors or activators. The

136 modularity produced by describing inhibition in this fashion also provides a base equation that

137 can be expanded to explain more complex kinetic interactions.

\section{Empirical modeling of complex inhibition kinetics}

139 By recognizing that changes in enzyme kinetic parameters are linked to the mass binding of the

140 inhibitor to the enzyme population, more complex situations can be rationalized and modeled in a

141 simple logical fashion. For example, one of the current targets for Alzheimer's disease treatment

142 is $\gamma$-secretase, an enzyme that cleaves the $\mathrm{C}$ terminal of the amyloid precursor protein. This

143 cleavage leads to the production of (1-X) amyloid fragments which results in the formation of the

$144 \beta$-amyloid peptides (1-40) and (1-42) (Burton et al., 2008). The formation of $\beta$-amyloid (1-42) is

145 highly associated with the pathology of Alzheimer's disease (Burton et al., 2008). Cellular

146 models of amyloid production, usually, rely on the expression of the 99-aminoacid C-terminal

147 fragment of amyloid precursor protein, known as C99, as a model substrate for $\gamma$-secretase. One

148 of the drug candidates that had been investigated using this cellular model is DAPT ( $\mathrm{N}-[\mathrm{N}-(3,5-$

149 difluorophenacetyl)-L-alanyl]-S-phenylglycine t-butyl ester). DAPT is described as having

150 complex kinetic interactions with $\gamma$-secretase. At low amyloid precursor protein concentrations, 
151 low concentrations of DAPT have been observed to increase $\gamma$-secretase catalytic production of

152 amyloid- $\beta$ 1-40 and 1-42 (EC50 72 nM) (Svedruzic, Popovic \& Sendula-Jengic, 2013). However

153 as DAPT concentrations are increased the activation turns into inhibition (IC50 approx $140 \mathrm{nM}$ )

154 (Burton et al., 2008; Svedruzic, Popovic \& Sendula-Jengic, 2013). At higher concentrations of

155 substrate DAPT only appears to inhibit the enzyme (IC50 approx $20 \mathrm{nM}$ ) (Burton et al., 2008).

156 These observations are further complicated by the substrate activation and inhibition of $\gamma$ -

157 secretase by amyloid precursor protein (Svedruzic, Popovic \& Sendula-Jengic, 2013). However,

158 these observations are all that is needed to produce a quick, simple empirical model of this

159 interaction. The fact that DAPT produces different effects at different amyloid precursor protein

160 concentrations indicates that the enzyme can be considered to have various forms, at least one at

161 low substrate concentration and one at higher concentrations. The presence of multiple

162 conformations is also supported by the apparent substrate activation of the enzyme at elevated

163 substrate concentrations and subsequent substrate inhibition as the substrate concentration is

164 further increased.

165 So to start with the substrate interactions can be modeled with an expansion of the Michaelis-

166 Menten equation that accounts for a change in the enzyme activity over increasing substrate

167 concentrations (equation 4) (Walsh, 2012).

$$
v=V_{1} \frac{[S]}{[S]+K_{1}}-V_{1} \frac{[S]}{[S]+K_{2}}+V_{2} \frac{[S]}{[S]+K_{2}}-V_{2} \frac{[S]}{[S]+K_{3}}
$$


169 At low substrate concentrations the substrate binding $\left(\mathrm{K}_{1}\right)$ can be described using a term that

170 mimics the Michaelis-Menten equation. At higher substrate concentrations the binding of an

171 additional substrate molecule $\left(\mathrm{K}_{2}\right)$ pushed the $\gamma$-secretase to an increased rate of substrate

172 hydrolysis $\left(\mathrm{V}_{2}\right)$. At even higher substrate concentrations $\left(\mathrm{K}_{3}\right)$ the $\gamma$-secretase activity is inhibited

173 by the substrate. However, fitting this equation (for examples see Kemmer \& Keller, 2010) to the

174 data clearly indicates that the changes in catalytic activity are too extreme to fit with the

175 hyperbolic Michaelis-Menten like curves. By adding Hill coefficients, a more sigmoidal change

176 in the data, induced by the substrate binding, can be modeled (equation 5).

$v=V_{1} \frac{[S]}{[S]+K_{1}}-V_{1} \frac{[S]^{H 1}}{[S]^{H 1}+K_{2}^{H 1}}+V_{2} \frac{[S]^{H 1}}{[S]^{H 1}+K_{2}^{H 1}}-V_{2} \frac{[S]^{H 2}}{[S]^{H 2}+K_{3}^{H 2}}$

177

178 With the enzyme described as different forms relating to substrate concentrations, interactions

179 with DAPT are readily defined. At high concentrations of substrate, DAPT acts as a regular

180 inhibitor of $\gamma$-secretase catalytic activity. Inhibitory changes in catalytic activity directly relate to

181 the fraction of the enzyme population bound by inhibitor as described by the mass action binding

182 term. The effect on the substrate affinity and catalytic activity are both dependent on the same

183 binding term, and the inhibition mirrors the inhibition produced by very high concentrations of

184 substrate (equations 6-7). The similarity between these interactions suggests that the inhibition

185 by substrate at higher concentrations may relate to additional substrate interactions with $\gamma$ -

186 secretase rather than through substrate aggregation. 


$$
V_{2}=V_{2}-\left(V_{2}-V_{2 i}\right) \frac{[I]^{H x x}}{[I]^{H x x}+K_{x x 1}^{H x x}}
$$

188

$$
K_{2}=K_{2}-\left(K_{2}-K_{2 i}\right) \frac{[I]^{H x x}}{[I]^{H x x}+K_{x x 1}^{H x x}}
$$

189 The effect on $\gamma$-secretase at low concentrations of amyloid precursor protein is only slightly more

190 complicated as it has to be described using two interactions between the enzyme and DAPT. In

191 an expansion of the inhibitory term, (equations 8-9), almost identical to the expansion of the

192 Michaelis-Menten equation above (equation 4), the stimulation at low concentrations of DAPT

$193\left(\mathrm{~V}_{1 \mathrm{is}}, \mathrm{K}_{1 \mathrm{is}}\right)$ and inhibition at high DAPT concentrations $\left(\mathrm{V}_{1 \mathrm{ii}}, \mathrm{K}_{1 \mathrm{ii}}\right)$ are both described

194 mathematically using mass action terms.

$$
V_{1}=V_{1}-\left(V_{1}-V_{1 i s}\right) \frac{[I]^{H x 1}}{[I]^{H x 1}+K_{x 1}^{H x 1}}+\left(V_{1}-V_{1 i s}\right) \frac{[I]^{H x 2}}{[I]^{H x 2}+K_{x 2}^{H x 2}}-\left(V_{1}-V_{1 i i}\right) \frac{[I]^{H x 2}}{[I]^{H x 2}+K_{x 2}^{H x 2}}
$$

$$
K_{1}=K_{1}-\left(K_{1}-K_{1 i s}\right) \frac{[I]^{H x 1}}{[I]^{H x 1}+K_{x 1}^{H x 1}}+\left(K_{1}-K_{1 i s}\right) \frac{[I]^{H x 2}}{[I]^{H x 2}+K_{x 2}^{H x 2}}-\left(K_{1}-K_{1 i i}\right) \frac{[I]^{H x 2}}{[I]^{H x 2}+K_{x 2}^{H x 2}}
$$


197 So empirically, without taking into account any information about the mechanism, a kinetic

198 equation which describes three enzyme states produced by substrate concentration and three

199 interactions between DAPT and $\gamma$-secretase can be described (Figs. 2-3). This equation allows

200 for the simple evaluation of DAPT as an inhibitor and provides insight into problems DAPT may

201 induce by increasing $\beta$-amyloid production at normal amyloid precursor protein concentrations.

202 This empirical approach also provides some perspective on the IC50 and EC50 values (Burton et

203 al., 2008; Svedruzic, Popovic \& Sendula-Jengic, 2013) described in the literature. At high

204 concentrations of substrate, DAPT is reported to have an IC50 around $20 \mathrm{nM}$, which would

205 suggest the DAPT is interacting with forms of $\gamma$-secretase predominately bound by two substrate

206 molecule $\left(\mathrm{K}_{\mathrm{xx} 1} 70 \mathrm{nM}\right)$. Elevated concentrations of substrate would occur in systems setup to

207 over express substrate. While the disassociation constant between the enzyme and inhibitor is

208 somewhat higher than the IC50 this inhibitory effect may be amplified by the inhibitory effects of

209 the third substrate binding interaction $\left(\mathrm{K}_{3} 605 \mathrm{nM}\right.$ expression plasmid). At lower substrate levels

210 DAPT (IC50 $140 \mathrm{nM}$ ) would be interacting with a $\gamma$-secretase population fluctuating between

211 enzymes interacting with a single substrate $\left(\mathrm{K}_{\mathrm{x} 2} 553 \mathrm{nM}\right)$ or two $\left(\mathrm{K}_{\mathrm{xx} 1} 70 \mathrm{nM}\right)$ producing an

212 apparent higher IC50 resulting from the mix. Additionally the EC50 for activation ( $72 \mathrm{nM})$ falls

213 very close to the disassociation constant for DAPT induced stimulation (Kx1 $30 \mathrm{nM})$. Using

214 these models allows for facile hypothesis generation, such as, a dimmer of DAPT may be a

215 practical way to eliminate the activation of $\gamma$-secretase produced by lower concentrations of

216 DAPT.

217 A comparison of the work involved in producing the conventional mechanistic model (equation

218 10) (Svedruzic, Popovic \& Sendula-Jengic, 2013) versus the empirical approach described here

219 also highlights the utility of this approach. Equation 5 and the expansions used to describe the 
220 interactions of DAPT with $\gamma$-secretase were produced, analyzed and fit to the data (see

221 supplementary information for fitting) in a few hours using the solver feature of excel (Kemmer

$222 \&$ Keller, 2010). The classical mechanistic approach used to describe the same kinetic process

223 involved the generation of a complex reaction schematic with 14 enzyme, substrate and inhibitor

224 interactions (Svedruzic, Popovic \& Sendula-Jengic, 2013). This reaction scheme was used to

225 define around 25 disassociation constants and three rate constants. The equation derived from

226 this structure was constructed from a connection matrix which was then fed into Mathematica to

227 produce a simplified version that ultimately only had five kinetic constants, (equation 10). While

228 equation 10 does contain fewer parameters than the 17 constants used in equation 5 , a

229 comparison of the predicted values produced using equation 10 with the observed experimental

230 data suggests that equation 10 does not fit the data very well (Fig. 2). Refitting the parameters of

231 equation 10 only marginally improved the models ability to fit the observed data (Fig. 4).

232

$v=\frac{\left.\left.V_{1}[S]\left(\frac{1}{1+\frac{[S]}{K_{s i}}}\right) \frac{1}{1+\frac{[I]}{K_{i i}}}\right)+V_{2}[S]\left(\frac{1}{1+\frac{[I]}{K_{i i}}}\right) \frac{1}{1+\frac{[I]}{K_{i a}}}\right)}{\left.[S]\left(\frac{1}{1+\frac{[S]}{K_{s i}}}\right)+K_{0.5 s}\left(\frac{1}{1+\frac{[I]}{K_{i i}}}\right) \frac{1}{1+\frac{[I]}{K_{i a}}+\frac{[I]}{K_{s i}}}\right)}$

233 Boxplots and correlation plots were used to evaluate the fit associated with each model. Ideally a

234 correlation plot of calculated versus observed data should produce a slope of one (Fig. 4) and an

$235 \mathrm{R}^{2}$ as close to one as possible, providing a visualization of the models fit. The residual boxplot 
236 provided a similar representation, where improvements in fitting of the models were evaluated

237 based on decreases in spread and increased symmetric distribution around zero. The correlation

238 plot produced by equation 10 (Fig. 4A) suggested that it was able to approximate the data fairly

239 well. However, the boxplot produced a negative asymmetric distribution of the residuals (Fig.

240 5A). Refitting the kinetic parameters associated with equation 10 improved the slope of the

241 correlation plot (Fig. 4B) and also improved the symmetric distribution of the residuals around

242 zero (Fig. 5B). Equation 5 however improved both the slope and the $\mathrm{R}^{2}$ value for the correlation

243 (Fig. 4C). A marked improvement in the symmetry and spread of the residual values was also

244 observed (Fig. 5C). However, as previously mentioned equation 10 only relies on five kinetic

245 parameters while equation 5, when expanded to describe DAPT interactions, contains 17 . Thus,

246 an increase in kinetic parameters might be viewed as over fitting of the data as models of greater

247 complexity are known to produce improved fitting (Burnham and Anderson, 2002). To evaluate

248 whether the improvement in fitting provided by equation 5 resulted from over fitting, equation 5

249 and 10 were compared using the bayesian information criterion (BIC). BIC was developed to

250 specifically penalize increasing complexity in model selection where a difference greater than ten

251 is considered strong evidence against the higher value (Burnham \& Anderson, 2002; Faraway,

252 2004). Not surprisingly when the BIC was used to compare equation 10 with the results

253 produced by refitting the kinetic constants of equation 10 (Fig 4A-B), a significant improvement

254 in the BIC value was observed (published values $\mathrm{BIC}=597$, refit kinetic parameters BIC 515).

255 The decrease in BIC was attributed to a reduction in residuals without any increase in the

256 complexity of equation 10 . Examination of the $\mathrm{BIC}$ value produced by equation $5(\mathrm{BIC}=448)$

257 also suggested a significant improvement over the fit achieved with equation 10 even though the

258 number of parameters had increased by 12 . 
259 The improper fit produced by equation 10 may relate to several factors, such as a lack of hill

260 coefficients in the equation. Even though, individual hill coefficients were used in the initial

261 description of the raw experimental data (Svedruzic, Popovic \& Sendula-Jengic, 2013) they were

262 left out of the simplified form of the equation ultimately published. Additionally, equation 10

263 only models C99-APP expression. The equation does not take into account normal cell line

264 amyloid precursor protein expression levels, i.e. the substrate concentration is defined as the

265 amount of vector used to produce the substrate and excludes endogenously produced substrates.

266 However, neither of these things can be easily evaluated due to the confusing notation of the

267 equation that includes terms that do not distinguish between binding and effect. In addition, the

268 paper lacks a proper overlay of the experimental data with the model produced using equation 10.

\section{Concluding remarks}

270 The information that can be obtained through proper kinetic modeling is invaluable for the

271 understanding of processes at the molecular level. The way mechanistic equations are currently

272 developed obscures the relationships between binding constants and effect on the enzyme. This

273 lack of utility and obscurity has led to the marginalization of the enzyme kinetic field in a decade

274 where tremendous amounts of money are being funneled into understanding biology at the

275 molecular level. While the improper use of kinetic models in drug development and the study of

276 biological processes may not be the primary cause of the problems plaguing the biological

277 sciences (Wadman, 2013) it is contributing to them. This is exemplified by the development of

278 DAPT as a $\gamma$-secretase inhibitor. Elan Pharmaceuticals rather than doing a thourough

279 characterization used the standard IC50 method (Dovey et al., 2001). The value they reported

280 (IC50 $20 \mathrm{nM}$ ) suggested they only looked at DAPT's effect at higher substrate concentrations 
281 (Burton et al., 2008). The results, more than a decade of research into a compound that holds

282 very little promise of ever being useful in the treatment of Alzheimer's disease. While DAPT

283 never made it to clinical trials and our understanding of $\gamma$-secretase benifited from its study, the

284 kinetic models used to describe DAPTs effect potentially delayed appropriate insights by a

285 decade. Indeed based on these inhibitors new classes of $\gamma$-secretase modulators are being

286 developed as potential therapeutics for Alzheimer's disease. However, characterization of their

287 efficacy are once again relying on IC50s and EC50s (Mitani et al., 2012). The failings of $\gamma$ -

288 secretase inhibitors clearly demonstrate that when assessing the effect of compounds on $\gamma$ -

289 secretase activity it cannot be taken for granted that the effect will stay the same over varying

290 substrate concentrations. The development of DAPT as a $\gamma$-secretase inhibitor only hints at the

291 enormity of time, money and resources that have been lost as a result of the marginalization of

292 enzyme kinetic in favor of simplified inhibition models or IC50s.

293 The failure of Alzheimer's disease drug candidates have been attributed to many factors such as

294 the initiation of clinical trials without proper insight into therapeutic mechanisms, improper

295 design of the studies and a lack of mechanistic understanding of the disease itself (Becker et al.,

296 2014; Schneider et al., 2014). To address these issues Becker et al., 2014, have stated that the

297 development of sound scientifically grounded mechanistic theories of disease progression needs

298 to be a priority. While it is easy to agree with these ideas, the persistent use of inappropriate

299 kinetic models, which mask more complex molecular interactions, will continue to obscure both

300 disease mechanism and potential therapeutic intervention.

\section{List of Supplementary Materials}


302 Data fitting in excel: secretase supplementary.xls

303 Key defining the kinetic constants of Equation 5

\section{Acknowledgements}

305 I would like to thank Dr. Svedružić for kindly sharing the raw data from his study on DAPT and $306 \gamma$-secretase interactions.

\section{References}

308 Becker RE, Greig NH, Giacobini E, Schneider LS, Ferrucci L. 2014. A new roadmap for drug 309 development for Alzheimer's disease. Nature reviews. Drug discovery 13:156.

310 Bernett MJ, Blaber SI, Scarisbrick IA, Dhanarajan P, Thompson SM, BlaberM. 2002. Crystal 311 structure and biochemical characterization of human kallikrein 6 reveals that a trypsin-like 312 kallikrein is expressed in the central nervous system. The Journal of Biological Chemistry $313 \quad 277: 24562-24570$.

314 Burnham KP, Anderson DR. 2002. Model selection and multimodel inference: a practical 315 information-theoretic approach. Springer.

316 Burton CR, Meredith JE, Barten DM, Goldstein ME, Krause CM, Kieras CJ, Sisk L, Iben LG,

317 Polson C, Thompson MW, Lin XA, Corsa J, Fiedler T, Pierdomenico M, Cao Y, Roach AH, 318 Cantone JL, Ford MJ, Drexler DM, Olson RE, Yang MG, Bergstrom CP, McElhone KE, 319 Bronson JJ, Macor JE, Blat Y, Grafstrom RH, Stern AM, Seiffert DA, Zaczek R, Albright CF, 
320 Toyn JH. 2008. The amyloid-beta rise and gamma-secretase inhibitor potency depend on the

321 level of substrate expression. The Journal of biological chemistry 283:22992-23003.

322 Dodgson KS, Spencer B, Williams K. 1956. Examples of anti-competitive inhibition. Nature $323 \quad 177: 432-433$.

324 Dovey HF, John V, Anderson JP, Chen LZ, de Saint Andrieu P, Fang LY, Freedman SB, Folmer B, 325 Goldbach E, Holsztynska EJ, Hu KL, Johnson-Wood KL, Kennedy SL, Kholodenko D, Knops 326 JE, Latimer LH, Lee M, Liao Z, Lieberburg IM, Motter RN, Mutter LC, Nietz J, Quinn KP, 327 Sacchi KL, Seubert PA, Shopp GM, Thorsett ED, Tung JS, Wu J, Yang S, Yin CT, Schenk DB, 328 May PC, Altstiel LD, Bender MH, Boggs LN, Britton TC, Clemens JC, Czilli DL, Dieckman329 McGinty DK, Droste JJ, Fuson KS, Gitter BD, Hyslop PA, Johnstone EM, Li WY, Little SP, 330 Mabry TE, Miller FD, Audia JE. 2001. Functional gamma-secretase inhibitors reduce beta331 amyloid peptide levels in brain. Journal of Neurochemistry 76:173-181.

332 Faraway JJ. 2004. Linear Models with R. Chapman \& Hall/CRC, Boca Raton, FL.

333 Fontes R, Ribeiro JM, Sillero A. 2000. Inhibition and activation of enzymes. The effect of a 334 modifier on the reaction rate and on kinetic parameters. Acta biochimica Polonica 47:233-257.

335 Kemmer G, Keller S. 2010. Nonlinear least-squares data fitting in Excel spreadsheets. Nature 336 Protocols 5:267-281.

337 McElroy WD. 1947. The mechanism of inhibition of cellular activity by narcotics. The Quarterly 338 Review of Biology 22:25-58.

339 Mitani Y, Yarimizu J, Saita K, Uchino H, Akashiba H, Shitaka Y, Ni K, Matsuoka N. 2012.

340 Differential effects between $\gamma$-secretase inhibitors and modulators on cognitive function in 341 amyloid precursor protein-transgenic and nontransgenic mice. The Journal of neuroscience $342 \quad 32: 2037-2050$.

343 Schneider LS, Mangialasche F, Andreasen N, Feldman H, Giacobini E, Jones R, Mantua V, 344 Mecocci P, Pani L, Winblad B, Kivipelto M. 2014. Clinical trials and late-stage drug 
345 development for Alzheimer's disease: an appraisal from 1984 to 2014. Journal of internal

346 medicine 275:251-83.

347 Segel IH. 1975. Enzyme Kinetics: Behavior and Analysis of Rapid Equilibrium and Steady-State

348 Enzyme Systems. Wiley.

349 Sousa MO, Miranda TL, Costa EB, Bittar ER, Santoro MM, Figueiredo AF. 2001. Linear

350 competitive inhibition of human tissue kallikrein by 4-aminobenzamidine and benzamidine

351 and linear mixed inhibition by 4-nitroaniline and aniline. Brazilian Journal Of Medical and

352 Biological Research 34:35-44.

353 Spitzer M, Wildenhain J, Rappsilber J, Tyers M. 2014. BoxPlotR: a web tool for generation of 354 box plots. Nature Methods 11:121-122.

355 Svedruzic ZM, Popovic K, Sendula-Jengic V. 2013. Modulators of gamma-secretase activity can

356 facilitate the toxic side-effects and pathogenesis of Alzheimer's disease. PLoS One 8:e50759.

357 Wadman M. 2013. NIH mulls rules for validating key results. Nature F500:14-16.

358 Walsh R. 2012. Alternative Perspectives of Enzyme Kinetic Modeling. In: Ekinci D. ed.

359 Medicinal Chemistry and Drug Design. InTech. 357-372.

360 Walsh R, Martin E, Darvesh S. 2011. Limitations of conventional inhibitor classifications.

$361 \quad$ Integrative biology 3:1197-1201.

362 Yoshino M. 1987. A graphical method for determining inhibition parameters for partial and 363 complete inhibitors. Biochemical Journal 248:815-820. 


\section{Figure 1}

Classic inhibition schemes.

The A) Competitive B) Noncompetitive and C) Mixed-noncompetitive equations and the reaction schemes used to derive them. In the reaction schemes (E) equals enzyme, (S) equals substrate, (I) equals inhibitor and (P) equals the product. In the equations $(\mathrm{V})$ is equal to the velocity of the reaction, (V1) is the maximum velocity usually denoted as Vmax, (K1) is the substrate binding constant commonly denoted as the Km (Michaelis-Menten substrate affinity constant) and ( $\mathrm{Ki})$ is the inhibitor binding constant.

A

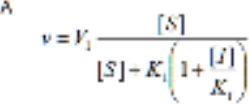

, $v=V_{1} \frac{[S]}{\left\lfloor[5]+K_{i}\left(1+\frac{[l]}{K_{i}}\right)\right.}$

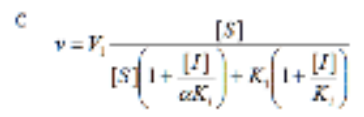

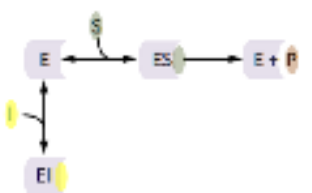
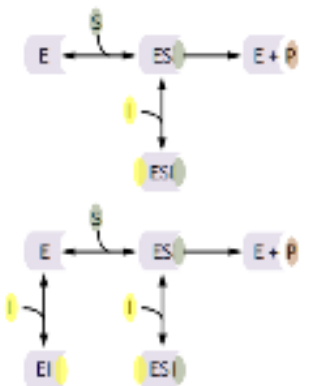
Figure 2

Modulation of $\mathrm{\gamma}$-secretase by DAPT.

Fitting of A) the mechanistic equation (Equation 10), B) Equation 10 refit and C) the proposed empirical equation (Equation 5) to the raw data for DAPT and $\gamma$-secretase interactions. Each Line represents a different concentrations of the amyloid precursor protein C-terminal fragment 99, expression vector (Svedruzic, Popovic \& Sendula-Jengic, 2013) 
PeerJ Reviewing Manuscript
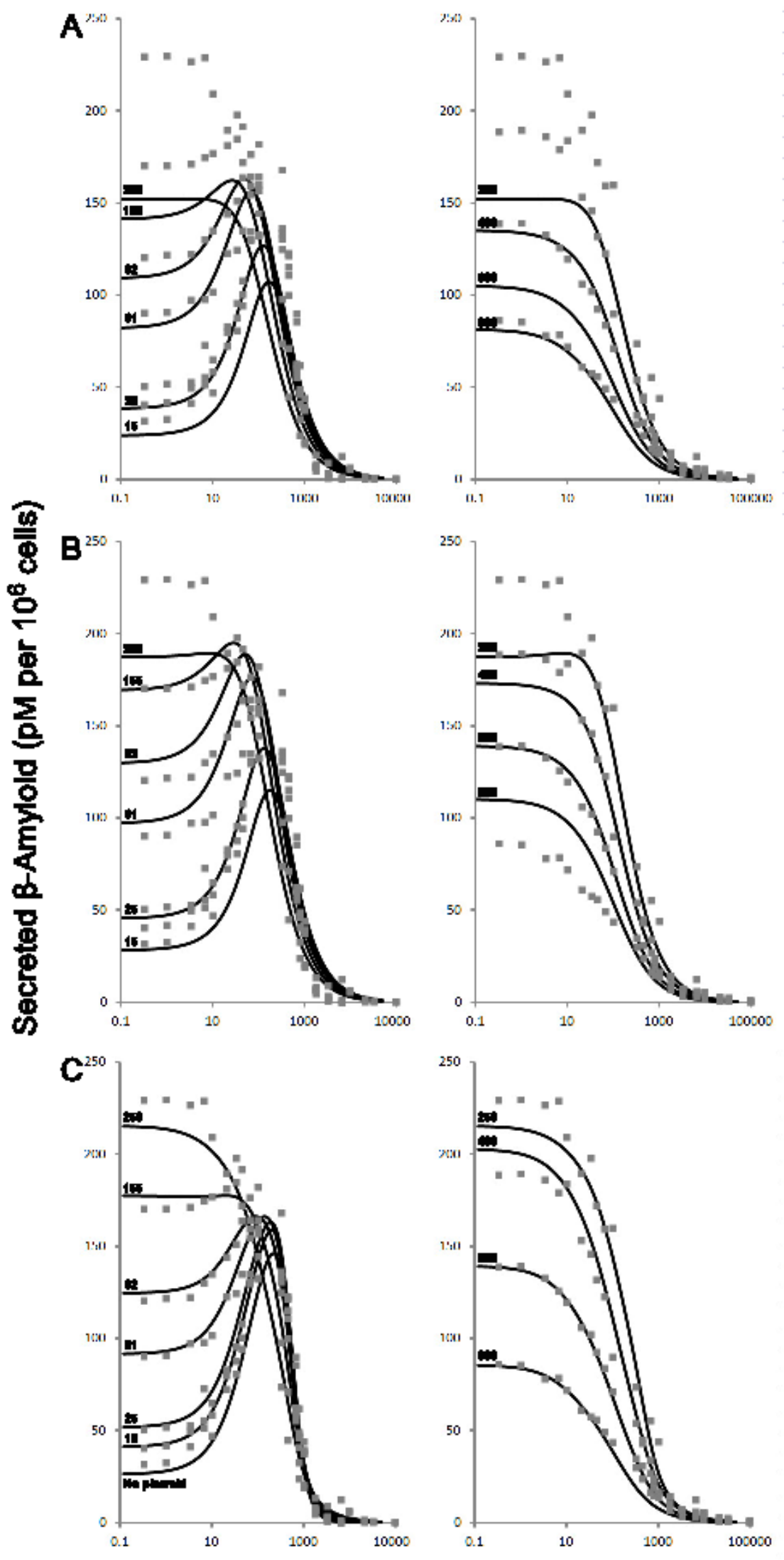

[DAPT] nM

PeerJ reviewing PDF | (v2014:09:2688:1:1:ACCEPTED 12 Oct 2014) 


\section{Figure 3}

Schematic of the interactions between $\gamma$-secretase, its substrate APP and DAPT.

The catalytic hydrolysis of APP is controlled by the number of molecules interacting with $\gamma$ secretase. Secondary binding of APP or DAPT increases the potential hydrolytic rate dramatically. However, interactions of a third APP or DAPT molecule shuts $\gamma$-secretase off suggesting the enzyme may become clogged or be highly regulated catalytically.

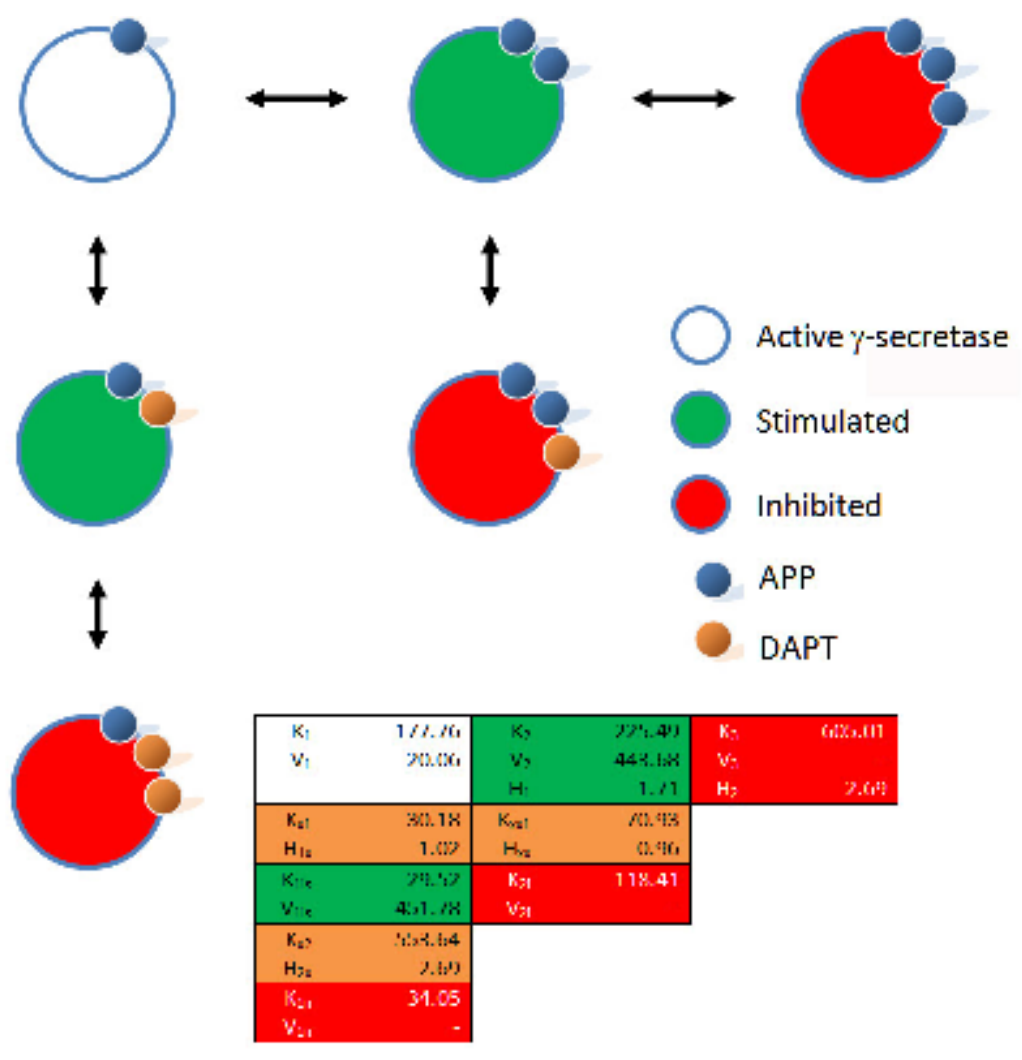




\section{Figure 4}

Global equation fitting to the experimental data.

Correlation plots of experimentally observed reaction rates versus calculated values obtained using A) equation 10 with the reported kinetic constants (correlation $r=0.968$ ), B) equation 10 with kinetic constants optimized in excel $(r=0.972)$ and $C)$ equation $5(r=0.993)$.
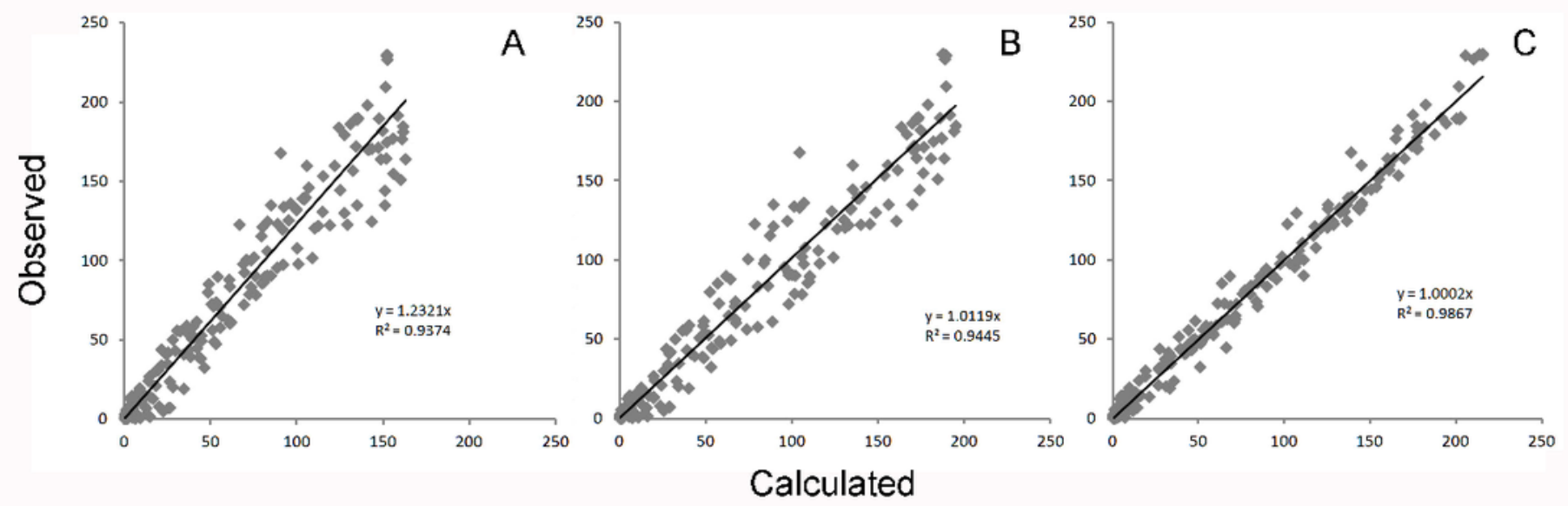
Figure $\mathbf{5}$ (on next page)

Boxplot of the residuals associated with each data fitting.

A) Residuals produced by equation 10 with the published values, B) residuals associated with the equation 10 after it was refit to the data and C) residuals associated with equation 5. Center lines show the medians; box limits indicate the 25th and 75th percentiles as determined by R software; whiskers extend 1.5 times the interquartile range from the 25th and 75th percentiles; outliers are represented by dots. Since equation 10 was not fit to background substrate concentrations, for $A$ and $B, n=208$, while for $C$ (Equation 5) $n=230$ sample points. This plot was generated using the web-tool BoxplotR (Spitzer et al., 2014). 


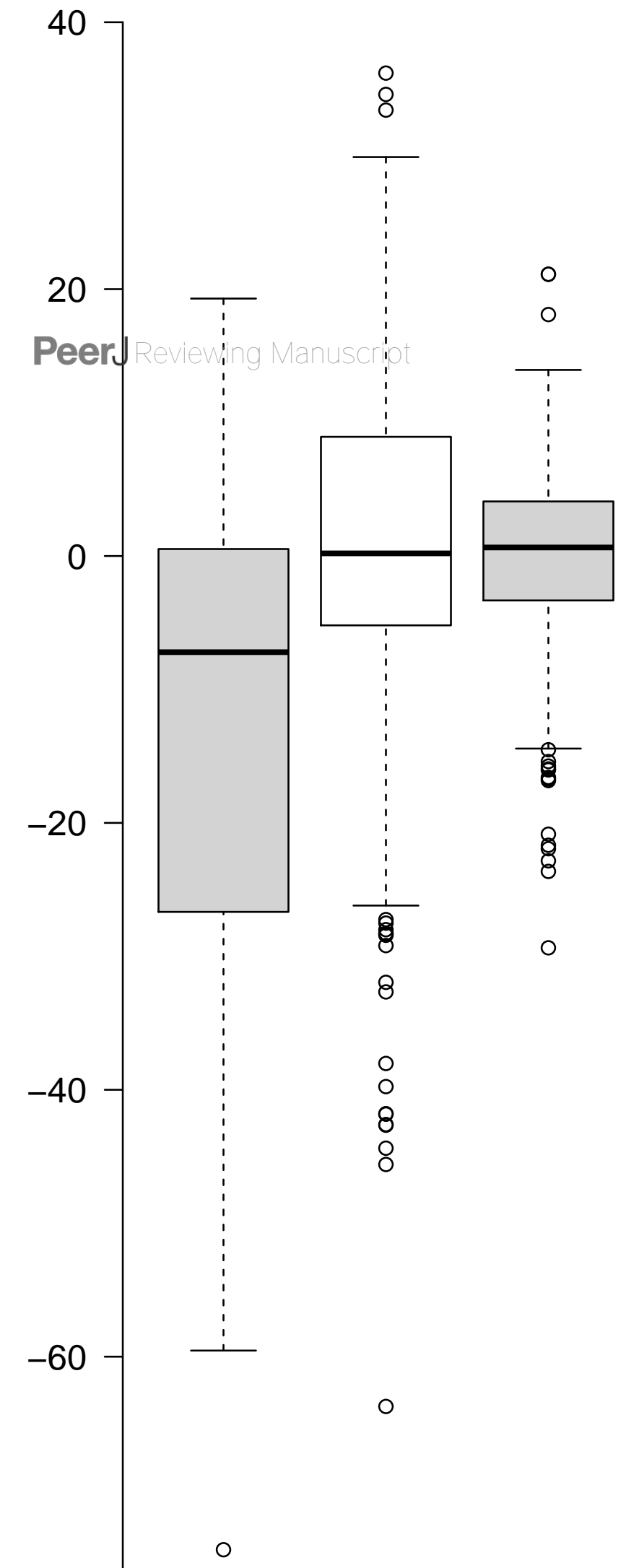

Peer) reviewing PDF | (v2014:09:2688:1:1:ACKEPTED 12 Oct 14) $-80$

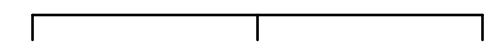

A $B \quad$ C

